\title{
Carnets
}

Revue électronique d'études françaises de l'APEF

Deuxième série - 5 | 2015

Imaginaires de guerre et autres conflits

\section{Les deux corps de Jules Romains}

Écrire la guerre quand on ne l'a pas faite

\section{Augustin Voegele}

\section{(2) OpenEdition}

Journals

Édition électronique

URL : http://journals.openedition.org/carnets/432

DOI : $10.4000 /$ carnets.432

ISSN : 1646-7698

Éditeur

APEF

Référence électronique

Augustin Voegele, "Les deux corps de Jules Romains », Carnets [En ligne], Deuxième série - 5 | 2015, mis en ligne le 30 novembre 2015, consulté le 30 avril 2019. URL : http://journals.openedition.org/ carnets/432; DOI : 10.4000/carnets.432

Ce document a été généré automatiquement le 30 avril 2019.

\section{(c) (i) \&}

Carnets est mis à disposition selon les termes de la licence Creative Commons - Atribution - Pas d'utilisation commerciale 4.0 International. 


\title{
Les deux corps de Jules Romains
}

\author{
Écrire la guerre quand on ne l'a pas faite
}

\section{Augustin Voegele}

1 Jules Romains, quoiqu'il soit resté loin du feu, a voulu témoigner de la Grande Guerre. Il a lutté contre l'événement en cours dans un poème, Europe. Il a lutté contre la Seconde Guerre qui s'annonçait, qui se préparait, dans des essais, dans des discours - mais aussi loin du regard indiscret, inutile, du public, en conseillant les puissants. Dans Les Hommes de bonne volonté, il pouvait donc parler, légitimement, de l'arrière - du courage discret de certains, des menues lâchetés ouvrières, de l'héroïsme oratoire des discoureurs. Mais il a su aussi - parce qu'il s'est documenté, et parce qu'il était doué de cette empathie qui fait les vrais poètes et les grands romanciers - raconter l'épopée misérable de Verdun, et faire entendre, plus puissants qu'aucune rhétorique, le vacarme du canon et les hurlements des soldats.

\section{L'engagement pacifiste de Jules Romains}

2 Jules Romains n'a pas été au front: pendant la première Guerre mondiale, il occupe « un obscur poste à l'arrière " (Robert, 2002: 89), et il passe la Seconde à New-York et à Mexico. Cela ne l'a pas empêché d'être engagé dans une guerre sans merci : celle que les amoureux de la paix de tous bords ont menée contre la guerre ${ }^{1}$.

3 Après les espoirs du siècle naissant, après cette « époque qui avait bien des raisons de ne pas être mécontente d'elle-même, et de croire à l'avenir » (Romains, 1958 : 15), la Grande Guerre est un cataclysme presque improbable. Jules Romains se le jure: il fera tout, désormais, pour empêcher la guerre, pour sauver l'Europe, «la plus belle [maison] du monde (...), la plus glorieusement habitée par l'esprit (...), la plus forte de ressources », des «forces mauvaises [qui] travaill[ent] à la déchirer " (Romains, 1946b : 289) - car pour lui, la guerre mondiale est avant tout européenne, et c'est au plus intime de l'Europe qu'il choisit de saisir l'heure suprême qui précède et précipite la catastrophe :

Et c'est pourquoi le premier août à quatre heures et demie du soir, Jean Jerphanion, Français de vieille souche et homme d'Europe entre autres, qui se trouvait avec sa jeune femme dans un champ à côté de l'oncle Crouziols, entendit la cloche de Saint- 
Julien-Chapteuil, canton d'entre les cantons, sonner pour les paysans de ce terroir antique le tocsin de la mobilisation (Romains, 1937a : 294).

Le voilà donc occupé à courir les antichambres, à jouer aux diplomates de l'ombre, il n'hésite à fréquenter personne, il rencontre Henri de Man, se met au service de la bonne volonté (malheureusement éphémère) du jeune Léopold III de Belgique, il est accueilli en très grande pompe par l'Allemagne nazie, et Goebbels lui envoie à Paris un " émissaire " pour s'excuser auprès de lui d'avoir confié la traduction allemande des premiers tomes des Hommes de bonne volonté à un "Juif », Franz Hessel, « l'auteur de la seconde traduction de Proust » (Romains, 1940 : 267-268)... En d'autres termes, Romains ne recule devant rien pour sauver la paix : il est prêt à souiller son nom devant la postérité, à se compromettre avec les moins fréquentables de ses contemporains - non par lâcheté, non par vanité, mais bien par esprit de sacrifice, et par volonté de sauver la civilisation et ses contemporains d'un nouveau conflit mondial qu'il prévoyait (à raison, hélas) plus meurtrier encore que le précédent.

5 Il se prodigue, et il prodigue les essais, discours, articles en faveur de la paix : c'est la part la plus ignorée de son œuvre (Romains n'avait besoin de personne pour défendre sa gloire romanesque et dramatique, André Figueras s'est chargé de veiller à sa renommée poétique (Figueras, 1952), mais personne encore n'a défendu l'essayiste politique qu'il était). Il combat " pour l'esprit et la liberté » (Romains, 1937b), il s'engage pleinement dans les "problèmes d'aujourd'hui» (Romains, 1931), il multiplie les «messages aux Français » (Romains, 1941), et leur crie à l'oreille : « Cela dépend de vous ! » (1939c).

6 Il travaille aussi à renforcer les institutions qui lui semblent en mesure de préserver l'Europe de la tentation du gouffre: il prend la présidence du PEN club international (1936-1939), honneur dont il se montre digne par anticipation, puisque, lors du congrès annuel de 1933 à Dubrovnik, il fait exclure, alors qu'il n'est pas même encore président de la section française, «la délégation allemande, coupable de 'collaboration avec le crime' et qui n'était composée que d'écrivains nazis ${ }^{2}$ » (Bourin, 1961 : 191). Il se décide même, en 1946, à ne plus bouder le Quai Conti - le poète intransigeant qui ne pardonnait pas à l'Académie certaines élections ridicules et certaines exclusions absurdes ne pouvait pas ne pas céder à des considérations plus hautes encore, et c'est ainsi que Romains finit, par souci de consolider les assises morales et intellectuelles de l'Occident, par accepter de siéger parmi les Immortels : «[Les] victoires [justifiées] étaient trop rares, (...) ce qui devait finir (...) par me faire prononcer des vœux, dont il ne fallut rien de moins qu'une deuxième guerre mondiale pour me relever " (Romains, 1958 : 22). Mais les institutions existantes lui semblent insuffisantes, et il propose de créer un Conseil des Sages, qui serait le Collège suprême auquel toutes les nations auraient à rendre raison de leurs actes. Il rêve d'une "Assemblée générale des Sages» (Berry, 1959: 47) "tout à fait indépendant [e] des gouvernements, opérant sur un plan supérieur à l'action politique immédiate» (Romains, 1955: 86), et «dont le pouvoir spirituel serait capable de préserver [l'humanité] contre les dangers qui la menacent » (Bourin, $1961: 217$ ).

7 La Grande Guerre, donc, a profondément bouleversé Romains, et l'a presque converti à une forme de conservatisme. Il prend conscience, devant le spectacle des ruines de l'Europe, du prix de ce qui dure et demeure. Il découvre de nouvelles valeurs, la permanence, la stabilité, la constance. Une certaine méditation qu'il met dans la bouche de son double fictionnel le plus fidèle (Jallez, l'enfant de Paris, normalien, agrégé de philosophie, écrivain, acharné défenseur de la paix au sein de la Société des Nations) en témoigne : 
Je (...) suis devenu plus sensible qu'avant la guerre à une certaine permanence dans les choses de la civilisation (...) Nous avons besoin de croire (...) à une espèce de durée végétale de la civilisation, à la propriété qu'elle a de se perpétuer malgré les accidents historiques, suivant des lignes de pousse qui dépendent d'un principe très fort, enveloppé dans le germe à l'origine (Romains, 1939b : 43).

\section{Agir en poésie}

8 Mais il ne pouvait suffire à Romains d'agir politiquement, diplomatiquement et institutionnellement. Il lui fallait agir en littérature, il lui fallait écrire un poème, puis un roman de la guerre. C'est chose faite avec Europe d'abord, avec Les Hommes de bonne volonté ensuite.

Europe paraît en plein cœur de l'événement, en 1916. Sa rédaction commence très peu après le début de la guerre :

Voilà soixante jours que l'Europe est en guerre,

L'Europe, mon pays, que j'ai voulu chanter (Romains, 1919: 9)

10 L'idéal continental et supranational est affiché dès les premiers vers, ainsi que la déception que constitue la guerre, après les rêves glorieux que les jeunes gens d'avant 14 avaient hérités du XIX ${ }^{\mathrm{e}}$ siècle, ce siècle béni et bienfaisant quoique agité :

Ce n'est pas ainsi que je rêvais

De commencer le chant de l'Europe (...),

Et je commence ta louange,

Europe, dans un grand tumulte ;

Je dis le chant de ta naissance

Dans le cri même de ta mort (Romains, $1919: 11$ ).

11 Puis Romains égrène comme les perles d'un chapelet les citadelles de la joie paisible : Londres, le Gothard, «la plaine de Picardie » (Romains, 1919: 33), Lyon, le Rhône, et « mille choses délicieuses » (Romains, 1919: 40), comme pour rappeler à l'Europe qu'elle pourrait être heureuse, si seulement elle savait jouir d'elle-même. Juxtaposer ainsi l'horreur et le bonheur, l'absurde et le paisible, c'est ce que d'autres ont fait, autour de lui et après lui - et notamment Thomas Mann, dans l'explicit de la Montagne magique, alors qu'il accompagne d'un œil attristé mais lointain Hans Castorp sur le champ d'honneur :

Ah, toute cette belle jeunesse avec ses sacs et ses baïonnettes, ses manteaux boueux et ses bottes! On pourrait avec une imagination humaniste et enivrée de beauté rêver d'autres images. On pourrait se représenter cette jeunesse: menant et baignant des chevaux dans une baie, se promenant sur la grève avec la bien-aimée, les lèvres à l'oreille de la douce fiancée, ou s'enseignant dans une aimable félicité l'art de tirer à l'arc. Au lieu de cela, elle est couchée, le nez dans cette boue de feu (Mann, 1931 : 973).

12 La baie lumineuse laisse la place à un torrent de pluie et de feu, de même que, dans Europe , les «choses divines » (Romains, 1919: 40) cèdent devant «la pluie [qui] tombe sur le cinq-centième jour de la guerre » (Romains, 1919: 43). Un poème ne sauvera pas l'Europe - à moins qu'il ne sache la réveiller de la meurtrière léthargie qui l'a saisie :

Europe ! Europe!

Je crie :

Ne te laisse pas mourir!

Cramponne-toi. Crispe-toi.

Reprends ta vie dans un spasme.

Écrase le dieu terrible ! (Romains, 1919 : 68) 
13 Et le poème se clôt sur un appel poignant et urgent, qui pourrait se résumer ainsi : "Européens de tous pays, unissez-vous». Dans une péroraison incantatoire, Romains convoque les foules européennes, il les met en demeure, il leur impose de prendre conscience de leurs pouvoirs, et de leurs responsabilités :

Foules du train et du théâtre,

Du café et du music-hall ;

Foule de Hyde-Park en Mai ;

Foule du Lido en Septembre;

Foules du port et du navire;

Foules de l'Europe vivante ;

Foules contraires à la mort ;

Je vous répète qu'il est temps (Romains, 1919 : 85).

\section{Avant l'événement, et après}

Quant aux Hommes de bonne volonté, dont la publication s'échelonne sur quinze ans (de 1932 à 1946), seuls deux tomes, c'est vrai, en sont consacrés à la Grande Guerre elle-même (ou plus exactement à la bataille de Verdun et à sa préparation), mais l'œuvre dans son ensemble se présente comme le tableau d'une longue « onde historique » dont la « crête » (Romains, $1964: 110$ ) est Verdun. Et il serait faux de dire que la guerre est absente des tomes qui précèdent Prélude à Verdun (XV) et Verdun (XVI), et de ceux qui les suivent. Dès le 6 octobre 1908, c'est-à-dire dès le tome inaugural, et dès l'incipit même, le spectre de la Guerre est présent :

Le mois d'octobre 1908 est resté fameux chez les météorologistes par sa beauté extraordinaire. Les hommes d'État sont plus oublieux. Sinon, ils se souviendraient de ce même mois d'octobre avec ferveur. Car il faillit leur apporter, six ans en avance, la guerre mondiale, avec les émotions, excitations et occasions de se distinguer qu'une guerre mondiale prodigue aux gens de leur métier (Romains, 1932: 26).

Déjà, la bravoure à distance, l'héroïsme loin du feu sont montrés du doigt - mais l'indolence aussi de tous ceux qui se montrent incapables de saisir la portée d'événements lointains et qui peuvent à tort sembler presque anodins. Car si Jules Romains a choisi le 6 octobre plutôt qu'un autre jour, c'est que cela lui permet de surprendre la réaction quelque peu nonchalante des Parisiens devant les événements de la veille : «Voilà qu'on nous apprend ce matin que la Bulgarie a proclamé son indépendance, hier 5 octobre. "Journée historique", constatent les manchettes de journaux. Ainsi, nous avons vécu, hier 5 octobre, une journée historique» (Romains, 1932: 28). Les treize tomes suivants montrent ceux qui seront qualifiés plus tard de «nobles de droit naturel» (Romains, 1946b : 64) - depuis l'instituteur Clanricard, qui décide de faire l'expérience des Loges, jusqu'à Laulerque, son rival en amour, qui, méfiant à l'égard de la maçonnerie, intègre une société secrète apparemment occupée à empêcher le conflit d'éclater - à la « recherche d'une Église » (Romains, 1934) au sein de laquelle ils pourraient travailler à construire le Temple de la bonne volonté. Cette angoisse idéale n'a d'ailleurs rien d'abstrait, et l'urgent prend parfois le pas sur l'intemporel. Il ne faut certes pas perdre de vue l'Orient moral vers quoi toutes les actions doivent converger; et précisément, le rôle des organisations souterraines est de réunir en faisceau les forces dispersées :

Si vous tâchez de regarder de près le mouvement de l'humanité depuis deux, trois, même quatre siècles, est-ce que vous n'êtes pas frappé par ce qu'il y a de nouveau?

(...) Eh bien, si vous sentez cela, vous sentez ce que les Maçons appellent la 
Construction du Temple. Je ne vous dis pas que tout le travail déjà fait par l'humanité dans ce sens, ce soient les Maçons qui l'aient fait. Non. Mais ils n'en ont jamais été absents. Et c'est bien eux qui dans la foule des travailleurs, sans cela trop éparse, trop facilement découragée, ont apporté le plan, la ténacité, la cohésion fraternelle (Romains, $1934:$ 294-295).

Toutefois, d'autres ligues secrètes, moins antiques, mais non moins actives, substituent aux «vues très générales, très vastes, [allant] dans le sens d'une transformation de l'humanité, d'une unification de l'humanité » des buts plus immédiats. Les dignitaires (invisibles, et dont on finit presque par douter qu'ils existent) de cette fédération insaisissable se convainquent qu'il faut « laisser de côté les grands projets d'avenir pour courir au plus pressé. Et que le plus pressé est d'éviter une guerre générale européenne » (Romains, 1934 : 63-64).

Quant aux onze derniers tomes, ils montrent comment, sur les décombres de l'Europe, prolifèrent les charognards qui s'acharnent à tirer profit du désastre, pendant que d'autres essayent de reconstruire la civilisation, malgré le deuil dont leur âme est alourdie. Car la victoire finale, "la plus grande en un sens, qu'il y ait jamais eue », ne pourra faire oublier à personne " qu'il n'y a jamais eu tant de morts » (Romains, 1939a : 240). « Le poids des morts grandit plus vite que la fierté de vainqueurs » (Romains, 1939a : 240), et, devant le spectacle du cénotaphe collectif des « morts pour la patrie » qui domine de son vide écrasant la Fête de la Victoire, Jerphanion ne peut se retenir de pleurer. La sonnerie aux morts, cette "sonnerie qui vous entre dans le torse, à la hauteur du diaphragme, comme une grande lame glacée " (Romains, 1939a: 243) continuera de retentir aux oreilles de l'Europe. C'est pourquoi d'ailleurs Romains montre ce vétéran de 14 qui se suicide, quinze ans après l'Armistice, sur la butte du Vauquois. Le souvenir du massacre ne quitte plus l'Europe, en proie au désir de mourir de ses propres mains, et qui ne supporte pas le spectacle de sa propre décomposition: «Le monde actuel me dégoûte. Ce n'est pas pour ce monde-là que nous avons tant souffert (...) et que tant de nos camarades sont morts » (Romains, 1946a : 119-120) - voilà ce qu'écrit le capitaine Imbard dans sa lettre d'adieu.

\section{Recréation ou témoignage?}

Évoquer la guerre elle-même, cependant, est délicat pour Romains : il n'a pas vécu dans les tranchées, et il n'aime guère les héros de l'arrière. George Allory, l'Académicien violeur des Hommes de bonne volonté, et son emphase guerrière n'ont rien de sympathique. Jerphanion s'emporte ainsi : « Hier encore, par exemple, j'ai lu un article de cette ordure de George Allory, sur Verdun! Sur 'la ruée de Verdun'! sur 'le poilu de Verdun'! (...) Il faudrait tuer des fantoches comme ça, les crever d'un coup de baïonnette, de cette baïonnette qui est si chère à leur éloquence " (Romains, 1938b:217). Et ce n'est pas Romains qui aurait écrit les douze tomes de L'Âme française et la guerre (Barrès, 1915-1920) - tout au contraire, il montre les soldats de Verdun en train de se passer

entre camarades, tantôt avec de grosses rigolades, tantôt avec des jurons de colère, les articles des Richepin, des Lavedan, des Barrès, et d'autres fiers-à-bras d'Académie, qui enseignaient aux hommes du front l'art de mourir, tout en les rassurant sur l'ennemi, piteusement armé, jobard et mourant de faim qu'ils avaient devant eux (Romains, 1938a : 79).

19 Le voilà donc embarrassé au moment de raconter Verdun. Se mettre dans l'esprit d'un général, cela lui est possible: il tient qu'un vrai romancier doit être capable de vivre, 
pendant le temps de la création, « une vie de notaire, de marchand d'étoffes, de prêtre » (Romains, 1952 : 47) ou de haut-gradé. Romains se vante d'ailleurs d'avoir été félicité par des militaires de haut prestige et de haute compétence. L'un d'entre eux lui aurait même déclaré : « 'Quand on m'envoie un nouvel officier, je commence par exiger qu'il lise votre Verdun » (Romains, $1964: 143$ ). Cela ne saurait toutefois le dispenser d'aborder la guerre de manière plus intime, de s'engager plus entièrement dans certains de ses personnages. Il n'est pas question pour lui de se glorifier, et d'envoyer Jallez à Verdun. Mais il a plusieurs frères fictionnels, et Jerphanion lui ressemble à la fois assez (il est un enfant du Velay, comme lui, et il est normalien lui aussi) et assez peu (puisqu'il finira ministre des Affaires étrangères) pour qu'il puisse le mobiliser sans honte, et profiter tout de même de sa ressemblance avec son créateur pour raconter la guerre de l'intérieur. Romains se scinde ainsi en deux, laissant à Paris son double séculier, et accompagnant son double spirituel à Verdun: le premier est l'individu Romains qui n'a pas connu la guerre, le second est Romains encore, mais cette fois le poète en qui la psyché universelle se cristallise.

Il reste le problème de la valeur du témoignage romainsien : quel crédit accorder à celui qui n'a pas fait l'expérience du feu ? Romains n'ignore pas qu'il est relativement mal placé (non pas moralement, mais bel et bien factuellement) pour évoquer les tranchées, et qu'il sera sans doute "dénié par les historiens comme source plausible pour l'histoire du front » (Robert, 2002: 89). Il sait aussi que l'image que lui-même se fait de la guerre ne peut être ni exacte, ni complète. Dans un chapitre clairement autoréflexif, intitulé «L'événement et son image » (Romains, 1938b : 43-45), il montre comment l'événement le plus atroce peut, au fil des "atténuations de texte", parvenir sous une forme très supportable à l'État-major. C'est la preuve qu'«il n'y a pas de fait matériel dont la matérialité soit assez dure pour que l'idée d'un homme tenace n'arrive pas à mordre dessus » (Romains, 1938b : 44). C'est pourquoi il se documente, et va consulter les sources les plus personnelles et les moins collectives, où il prend connaissance de ce qu'étaient la vie et la mort quotidiennes à Verdun, et en général sur le front, pour les soldats engagés au plus épais de la bataille :

Il n'a pas pris part à la guerre, mais il va sur le terrain, il regarde longuement à la Bibliothèque-musée de la guerre les objets exposés (...); il suit, de la conception à l'exécution, les ordres d'État-major (...); il consulte carnets de combattants, journaux de compagnies, s'initie à la psychologie du "poilu», à l'argot des tranchées, au vocabulaire de la guerre (Cuisenier, 1969 : 208).

21 Ce travail de renseignement n'a rien de distant, ou de froid, et les éléments collectés, Romains les fait passer par le filtre de son empathie d'écrivain. C'est ainsi d'ailleurs que ceux qu'on suit à la guerre lui ressemblent malgré tout: «Les principaux témoins romanesques de la guerre sont Clanricard et Jerphanion, c'est-à-dire deux intellectuels » (Touchard, 2002: 60) - ce qui ne signifie pas bien sûr que Romains ne se préoccupe pas des détails physiques de la guerre, et qu'il ne tente pas de transcrire les sensations mêmes de poilus qui luttent contre la boue. Ses dossiers préparatoires témoignent de ce souci de rendre palpable la souffrance physique des soldats :

boue et pieds gelés (...) sorte d'argile bleu-vert - se colle partout, visage, vêtements, mains - impossible à écoper. Plus on veut marcher, plus on enfonce. Si on reste immobile, fourmillements et brûlures dans les pieds. Æđèmes, pieds gonflés, violets, noirâtres, comme gangrène (Romains, $1982: 230$ ).

Le résultat semble convaincant, du moins aux yeux des écrivains. Roger Martin du Gard n'hésite pas à considérer Verdun comme un témoignage - preuve que Romains a su se 
dépouiller de son propre passé pour investir et revêtir la mémoire intime de l'Europe, alors même qu'il ne prétendait présenter qu'une "synthèse ", qu'une "recréation " (Robert, $2002: 90)$ : « Le grand témoignage qu'on attendait, le voilà (...) Maintenant, c'est fini (...) impossible d'y revenir après vous » (Romains, $1982: 155)$.

\section{Le front et l'arrière}

Romains ne se contente pas, en effet, contrairement à ce que laisse entendre Robert, de peindre la vie de l'arrière. Il n'est pas faux, mais pas exact non plus, d'affirmer que «Jules Romains fut (...) un observateur de la vie à Paris pendant la guerre », et que c'est «sans doute (...) d'abord de celle-ci qu'il rend compte » (Robert, 2002, 89). Certes, il montre comment s'organise loin du feu une vie parasitaire, dont les premiers bénéficiaires sont les aventuriers de la finance et les ouvriers mobilisés dans les usines - quoique son jugement sur les ouvriers profiteurs soit moins dépourvu d'indulgence que ne le laisse entendre Robert. Il est trop simple d'affirmer que, dans Les Hommes de bonne volonté, « la guerre révèle que le matérialisme ouvrier l'emporte sur l'idéalisme» (Robert, 2002 : 92). Romains ne montre à aucun moment l'ouvrier dans l'exercice d'une jouissance sans conscience: au contraire, ceux qui«tourne[nt] des obus » éprouvent "un bonheur ambigu » (Romains, 1938b : 243). Prenons Maillecotin, qui est depuis longtemps familier des lecteurs des Hommes de bonne volonté. Maillecotin est sympathique dans l'ensemble quoiqu'en temps de paix déjà, il manque quelque peu de rigueur morale, ou du moins syndicale; on le voit par exemple occupé à collaborer avec son patron, le fabriquant d'automobiles Bertrand:

Bertrand a fait placer, dans le couloir qui précède la caisse, une boîte aux lettres. Quand un ouvrier a l'idée d'une amélioration (...), il est prié de la mettre par écrit (... ). La semaine dernière, [Maillecotin] a déposé une nouvelle enveloppe (...). M. Bertrand va peut-être féliciter Maillecotin, ou l'interroger (...). Edmond se défend de le souhaiter trop fort. Même quand l'état de guerre n'est pas déclaré entre eux, les relations de l'ouvrier avec le patron doivent rester aussi impersonnelles et indirectes que possible. Le seul fait de suggérer, sur l'invite du patron, une amélioration possible, est déjà le témoignage d'un zèle bien suspect (Romains, 1935 : 40-41)

Il est vrai aussi que Maillecotin profite pleinement du luxe inhabituel que lui permet son salaire d'ouvrier de guerre. Il alimente les machines à mort, et c'est ainsi qu'il peut garnir somptueusement sa table, sur laquelle Romains dispose toutes les splendeurs de la conserve. Georgette et lui consomment des «boittes de conserve diverses, toutes de première qualité : boîtes de maquereau à l'huile, de thon, de saumon, de langouste ; boîtes de foie gras de chez Marie, de jambon de chez Olida ; terrines de mets préparés de chez Poinseul ( (Romains, 1938b : 244). Sa conscience, cependant, ne le laisse pas en paix (il s'avoue sourdement que «s'il fallait rattraper la bonne conscience, il y aurait beaucoup de chemin à faire » (Romains, 1938b : 248), et c'est avant tout la morale de classe qui est mise à mal. Si Maillecotin est saisi d'un certain malaise, c'est parce qu'il se trouve une ressemblance troublante avec les classes parasitaires dont il a toujours considéré (modérément, mais fermement) qu'elles étaient ce qu'il y avait de plus dangereusement inutile dans l'organisation d'une société :

Cette bâfrerie continuelle ne lui paraît pas très éloignée de ce qu'il détestait le plus chez les bourgeois d'avant-guerre. Et même, comme il est juste, il lui arrive de penser qu'elle est un peu plus répugnante par la coïncidence qu'elle offre avec 
certains événements, et par la façon dont se gagne l'argent qui la procure (Romains, $1938 b$ : 246). sépare la conscience de l'action. Pourtant, il lui arrive parfois de se confronter aux questions fondamentales, et de les aborder dans leur réalité nue, sans se laisser prendre aux jeux de la rhétorique ou du lyrisme :

Si on te disait : «Veux-tu que la guerre finisse demain; et qu'on ne se tue plus sur le front avec les obus que tu fabriques (...) même s'il faut que tu te remettes ensuite à travailler à dix, douze francs par jour?»... qu'est-ce que tu répondrais? Il s'interroge bien à fond, et il prononce: "Je répondrais: Oui, tout de suite.» (Romains, 1938b : 253)

Voilà pour l'arrière. Mais l'essentiel des volumes ne s'en passe pas moins du côté de Verdun et de la Butte du Vauquois. On retrouve dans ce récit de l'horreur du front la manière (pour ne pas dire parfois les manies) de Romains : l'« astronomique » précède le «moléculaire» (Cuisenier, 1954: 82), c'est une longue évocation unanimiste des mouvements et de la vie de cet individu collectif qu'est le «million d'hommes" (Romains, 1938a : 11) qui ouvre Prélude à Verdun; et Romains ne se prive pas d'introduire un peu de gaudriole au plus fort de la bataille - quand tel personnage occupé à admirer l'activité incessante d'un canon qui crache obus sur obus, sans jamais défaillir, se prend à éprouver une certaine jalousie fondée sur une association de pensée à la fois évidente et invraisemblable, compte tenu des circonstances. Voici les réflexions auxquelles Romains se laisse aller, et qu'il prête à un soldat, pour masquer ce qu'elles peuvent avoir d'inconvenant, eu égard non aux lois de la pudibonderie, mais à l'atrocité de la situation :

Notre admiration [devant un canon comme le 305] contient sûrement quelque chose de sexuel. Sans nous en rendre compte, nous somme extasiés, en tant que pauvres petits mâles, par un mouvement que nous reconnaissons, mais qui s'effectue à des dimensions surnaturelles; et aussi par cet organe miraculeux que sa détente apaise, sans l'abattre (Romains, 1938b : 261-262).

Romains glisse, de temps à autre, et au risque de paraître un peu léger, une note d'humour dans cette symphonie de la guerre. Au plus brûlant du combat, Fabre, le compagnon d'armes de Jerphanion, ressuscite une vieille scie: il déclare, d'un ton imperturbable, qu'il se tirera du déluge de feu « en se jetant dans les rangs de l'ennemi et en tranchant la discussion à l'arme froide " (Romains, 1938a : 78). Cette allusion aux ridicules théories militaires défendues avant-guerre par le Général Duroure n'est toutefois pas dépourvue de sérieux: car, si la scène comique est fictive, la sentence stratégique, elle, est empruntée à un ouvrage du général Foch, De la conduite de la guerre, publié en février 1904, et republié en 1915. Jules Romains aime en effet à laisser parler les documents : ils sont parfois les meilleurs témoins de la bravoure ou de l'incompétence des généraux - sans compter bien sûr qu'ils donnent à son récit cette caution de vérité que la biographie de l'auteur ne saurait lui fournir. Verdun se termine ainsi sur l'ordre du jour que Pétain adresse le 10 avril 1916 " aux troupes de la IIe Armée », et qui finit sur ces mots fameux : «Courage, on les aura! » (Romains, 1938b : 280)

\section{Alerter la foule, conseiller les puissants}

D'une façon plus générale, les Hommes de bonne volonté mêlent les personnages entièrement fictifs, les personnages à clef et les figures historiques : dans les deux tomes centraux, Duroure et Jerphanion (c'est-à-dire Foch et Romains) voisinent de la sorte avec 
Wazemmes et Fabre (qui ne sont que Wazemmes et Fabre) aussi bien qu'avec Pétain, Gallieni ou Joffre. Les personnages les plus intéressants sont sans doute les personnages intermédiaires, ceux qui n'appartiennent ni tout à fait à la réalité, ni tout à fait à la fiction, ceux qui permettent à Romains d'enter l'intime sur l'objectif. Nombreux sont ceux, en particulier, qui sont Romains sans être Romains, qui sont des presque-jumeaux, des frères ou des cousins lointains de l'auteur. L'instituteur Clanricard parlant de l'alarmante situation européenne à ses petits élèves, c'est un peu le père de Romains, ce discret instituteur parisien, et c'est bien sûr (avec un décalage d'une guerre mondiale) Romains lui-même, qui parle à ses lecteurs comme un maitre qui tente d'éveiller dans l'esprit de ses pupilles le sentiment du péril qui les menace, et avec eux toute la civilisation dont ils sont les enfants. Il règne en effet un silence dont l'inconscience est sans doute autant responsable que la nonchalance ou la lâcheté, et qu'il est urgent de briser :

" - Mes enfants, j'ai une chose à vous dire. Je ne sais pas si vos pères en parleront devant vous. L'autre jour, nous avons regardé ensemble la carte d'Europe (...) Vous vous rappelez : les Balkans sont ici. La Bulgarie, la Serbie, la Turquie, n'est-ce pas? Eh bien, il va probablement éclater une guerre par là, entre la Bulgarie et la Turquie. Et tous les gouvernements d'Europe sont liés de telle façon les uns aux autres par des traités d'alliance, par des conventions plus ou moins secrètes (...), qu'il se peut très bien que, si la guerre éclate, elle gagne toute l'Europe. » (Romains, $1932: 53-54)$

Cette contagion guerrière que les alliances rendent presque inévitable est sans doute ce qui inquiète le plus Romains dans la situation européenne : la paix est organisée de telle façon que le moindre conflit local se généralisera fatalement. Cette angoisse, cependant, n'empêche ni Romains ni Clanricard de se laisser captiver par moments par le charme robuste des armées - et ce que cette séduction peut avoir d'inavouable et de honteux contribue à la rendre troublante. Romains avouait avoir été «bouleversé [dans tous les sens du terme] par l'armée » (Romains, 1926 : 31), et Clanricard, le jour même de son prêche pacifiste, se laisse griser par la vitalité militaire :

Un escadron de dragons remontait la rue Custine (...) Clanricard, avec stupéfaction, se sentit parcouru d'une sorte de frisson délicieux. (...) Il se mit à vivre avec une intensité qui faisait que la substance de la vie devenait sensible tout entière, et que toute la masse de l'être vivant jouissait d'elle-même (Romains, 1932 : 172).

Il faut ainsi que l'esprit se déprenne de ce piège tendu par les apparences militaires : le bel ordre des casernes et des exercices ne préfigure en rien la réalité chaotique de la guerre.

31 Il y a ceux, donc, qui luttent discrètement, et dans la modeste mesure de leurs moyens, contre la guerre et ses séductions. Il y a ceux aussi qui conseillent les rois et les ministres, il y a les éminences grises qui travaillent à orienter la guerre, une fois qu'elle ne peut plus être empêchée. Maykosen, le conseiller intime de Guillaume II, est le double inversé et anticipé de Romains travaillant pour les intérêts de la paix et de la démocratie... Et si Maykosen est son jumeau en miroir, alors on peut penser que Romains ne se faisait pas trop d'illusions sur son influence auprès des puissants de ce monde. En effet, Guillaume II permet à Maykosen de "déraisonner ", il a le droit d'agiter des idées, et même de se montrer quelque peu familier avec l'empereur: «Maykosen s'avisa (...) que, sans le vouloir, il avait, pour s'adresser à son auguste ami, des façons plus libres, moins obséquieuses que par le passé ». Mais, si cela flatte sa vanité, c'est sans conséquences sur 
la marche des choses de la guerre : «L'Empereur avait écouté avec attention, même avec excitation. Mais il gardait un sourire d'incrédulité amusée » (Romains, 1938a : 215-220).

\section{Le physique et le moral}

Cependant, ces ruses, ces dispositifs que Romains agence pour s'infiltrer dans le monde de sa propre fiction, et pour introduire en retour ses personnages sans chair dans l'épaisseur physique de l'Histoire, ne sont pour lui que le moyen d'aborder, sans avoir l'air d'émettre abstraitement des hypothèses sociales, morales ou anthropologiques, la question qui le préoccupe entre toutes. Ce qui semble à Romains le plus désespérant, c'est la formidable régression morale que suppose et qu'entraîne la guerre: "Nous sommes en train de retrouver des états d'esprit du moyen âge : l'irrévérence pour la carcasse humaine et pour la chair vivante et morte » (Romains, 1938a : 181). Dès les Dossiers préparatoires, c'est un aspect de la guerre qui l'obsède: l' "indifférence totale à l'égard du corps vivant », le «mépris comique et macabre ( (Romains, $1982: 198)$ à son endroit lui semblent hideux. Toute la morale séculière qui avait permis à l'homme de sortir du moyen âge est anéantie d'un coup. La vie, qui était devenue la valeur suprême sur laquelle toute morale se fondait, n'est plus qu'une quantité négligeable :

Voilà, il me semble, la perte entre toutes irréparable. Il avait fallu à la civilisation des siècles de tâtonnements (...) pour apprendre aux hommes que la vie, la leur, celle des autres, est quelque chose de sacré. Tout ce travail est fichu. On ne s'en remettra pas (Romains, 1938a : 182).

Cela signifie que l'homme est moralement malléable au point qu'il n'est plus possible, dans quelque circonstance que ce soit, de lui faire confiance : «Nous savons maintenant qu'on peut faire faire aux hommes exactement n'importe quoi - et aussi bien avant qu'après cent ans de démocratie et dix-huit siècles de christianisme " (Romains, 1938a : 183). C'est pourquoi Romains consacre de nombreux chapitres à cette question qui l'obsède: comment peut-on amener un homme à accepter la guerre, et d'abord à supporter son horreur? Vivre dans la boue, et sous la menace permanente des obus, avec pour seule perspective d'être tué ou de voir mourir ses camarades, cela n'est possible que si l'on sait développer de tortueuses stratégies mentales. Jerphanion, ainsi, se donne le sentiment de ne rien risquer en s'inventant, à partir des éléments réels qui l'entourent, des abris chimériques (Romains, 1938a : 91-92). Mais il faut encore supporter l'idée de la durée du conflit. Il y a un moment où la consolation de la fatalité n'est plus assez puissante pour retenir le soldat sur la pente du désespoir ou de la révolte. Chacun se crée alors une idée sur laquelle il fixe son esprit, et qui lui permet de s'abstraire de la réalité du combat, ou de s'élever au-dessus de la mêlée : «l'idée qu'ils restent là (...) parce qu'il n'y a pas moyen de faire autrement ne suffirait pas à les soutenir (...) Alors chacun d'eux s'est procuré une suggestion personnelle (...) » (Romains, 1938b : 233). Jerphanion, par exemple, tente de s'hypnotiser en se convainquant de sa propre supériorité. Mais ni «la songerie, l'illusion de la sécurité personnelle ", ni l'idée fixe ne suffisent. Il faut aussi fortifier « l'illusion que le monde reste vivable (...)» (Romains, 1938a : 96), que la guerre peut être, aussi bien que la paix, le climat permanent de la vie. Jerphanion s'évertue donc à développer une vision: la guerre s'éternise, et les armées se défont, se dispersent, s'éparpillent en bandes. Il s'imagine lui-même menant une bande qui s'établirait dans la forêt du Meygal, et il trouve là la possibilité d'un bonheur précaire mais tout de même savoureux (Romains, 1938a : 97-101). 
Toutefois, après l' "enthousiasme des premiers jours" (Romains, 1938a : 169), le voici face à la certitude que « rien ne vaut ça » (Romains, 1938a : 171). Alors, dans ses lettres à Jallez, et au cours d'une promenade qu'ils font ensemble alors qu'il est en permission à Paris, il tente de répondre à la question essentielle : pourquoi les soldats, armés comme ils le sont, ne se révoltent-ils pas, qu'est-ce qui les pousse à rester sous le feu? Il y a cent facteurs qui se réunissent pour les empêcher de fuir, ou de retourner leurs armes contre ceux qui les envoient à la mort. D'abord, les états-majors ne relâchent jamais leur autorité, ne se laissent jamais aller à aucune mollesse ni faiblesse - et justement, «l'homme ne trouve le courage de se révolter que contre une autorité qui montre des signes de défaillance» (Romains, 1938b: 229). L'orgueil angoissé du mâle se joint également à la ligue des réflexes psychiques qui retiennent le soldat dans les tranchées : "Quand le poilu banal songe à se faire embusquer, il y a un argument qui l'arrête plus que tous les autres (...) Il se dit : 'Les femmes regardent... elles regardent si je pars aussi bien que les autres.' » (Romains, 1938 : 231). Mais, note André Cuisenier, « d'autres mobiles les poussent, aussi vieux que l'humanité : par exemple, un goût singulier du sacrifice » (Cuisenier, 1969 : 151). En effet, aux raisons négatives se joignent des instincts positifs sans compter qu'il se développe tout un imaginaire de la gloire militaire. Il y a notamment cette attirance presque irrésistible que l'homme du commun éprouve pour tout ce qui lui procurera des «thrills» (Romains, 1946c: 120): "J'ai l'impression que l'homme, quand sa raison ne le contrôle pas avec sévérité, résiste difficilement à l'attrait, à la promesse d'un grand choc émotionnel » (Romains, 1938b : 230-231). Et puis, Romains le note dans ses dossiers préparatoires déjà, l'homme du feu est libéré de toutes les tâches communes, sa vie est menacée par les obus peut-être, mais plus par la médiocrité. Comme le résume Cuisenier, « les combattants ont conscience d'habiter une région à part (...) Ils parcourent le monde avec un orgueil d'explorateurs » (Cuisenier, 1969: 151). Ils s'élèvent au-dessus du vulgaire, qu'ils regardent avec dédain. Le " guerrier débarrassé de travail et de soucis ordinaires " retrouve ainsi un "très antique état d'esprit» (Romains, 1982: 217) : il a «droit à être nourri et honoré » et «méprise le reste : femmes et esclaves » (Romains, $1982: 217$ ).

\section{Les discours et les cris}

Romains, ainsi, ne refuse pas le ton épique: mais ce que la guerre peut avoir d'apparemment grand, de superficiellement exaltant, n'est en vérité qu'illusion ou suggestion. Les envolées lyriques des patriotes de chambre qui confondent leur plume avec un fusil, et les joutes rhétoriques avec la guerre de tranchée, Romains les démythifie : la majesté de la guerre et la gloire du guerrier ne sont que des chimères que le soldat invente pour parer de couleurs grisantes sa misère, ou que ceux qui de loin décident de sa mort conçoivent pour l'aveugler. Et c'est en cela, peut-être, que la position de Romains (capable, grâce à son intuition empathique de romancier, d'être à la fois à Paris et à Verdun) est la meilleure, littérairement parlant : il entend à la fois les périodes des académiciens belliqueux et les cris des soldats, et les premières comme les seconds se retrouvent dans son roman, mis en perspective. Dans Les Hommes de bonne volonté, les harmonies oratoires ne cachent pas la stridence des obus, pas plus que le bruit du canon n'empêche d'analyser les détours meurtriers du discours patriotique, et de déjouer ses pièges. De la sorte, Romains, tout en racontant la guerre des soldats, défait la guerre des discoureurs, et c'est ainsi que, derrière le chant malgré tout épique qui célèbre, non pas 
Verdun, mais le courage et l'abnégation de ceux qui étaient à Verdun, on entend, discret parfois, mais souvent plus puissant que l'hymne, le contre-chant funèbre qui déplore et dénonce l'atrocité misérable de la guerre.

\section{BIBLIOGRAPHIE}

ANTOINE, Régis (2002). La Littérature pacifiste et internationaliste française, 1915-1935. Paris :

l'Harmattan.

BARRÈs, Maurice (1915-1920). L’Âme française et la guerre (11 volumes). Paris : Émile Paul.

BERRY, Madeleine (1959). Jules Romains. Paris : Éditions universitaires.

BOURIN, André (1961). Connaissance de Jules Romains. Paris : Flammarion.

CUISENIER, André (1954). Jules Romains et Les Hommes de bonne volonté. Paris : Flammarion.

CUISENIER, André (1969). Jules Romains : l'unanimisme et Les Hommes de bonne volonté. Paris :

Flammarion.

FIGUERAS, André (1952). Jules Romains. Paris : Seghers.

FOCH, Ferdinand ([1904], 1915). De la conduite de la guerre. Paris : Berger-Levrault.

MANN, Thomas ([1924], 1931). La Montagne magique [Der Zauberberg]. Traduit de l'allemand par

Maurice Betz. Paris : Fayard.

ROBERT, Jean-Louis (2002). « Verdun : le front et l'arrière. Jules Romains. », in Sophie Béroud, Tania

Régin (éds.). Le Roman social : littérature, histoire et mouvement ouvrier. Ivry-sur-Seine : Éditions de

l'Atelier, pp. 89-94.

ROMAINS, Jules ([1916], 1919). Europe. Paris : Gallimard.

Romains, Jules ([1908], 1926). La Vie unanime. Paris : Gallimard.

ROMAINS, Jules (1931). Problèmes d'aujourd'hui. Paris : Kra.

ROMAINS, Jules (1932). Le 6 octobre (Les Hommes de bonne volonté, Tome I). Paris : Flammarion.

ROMAINS, Jules (1934). Recherche d'une Église (Les Hommes de bonne volonté, Tome VII). Paris :

Flammarion.

ROMAINS, Jules (1935). Montée des périls (Les Hommes de bonne volonté, Tome IX). Paris : Flammarion.

RomAINS, Jules (1937a). Le Drapeau noir (Les Hommes de bonne volonté, Tome XIV). Paris :

Flammarion.

ROMAINS, Jules (1937b). Pour l'esprit et la liberté. Paris : Gallimard.

Romains, Jules (1938a). Prélude à Verdun (Les Hommes de bonne volonté, Tome XV). Paris :

Flammarion.

Romains, Jules (1938b). Verdun (Les Hommes de bonne volonté, Tome XVI). Paris : Flammarion. 
ROMAINS, Jules (1939a). Vorge contre Quinette (Les Hommes de bonne volonté, Tome XVII). Paris :

Flammarion.

Romains, Jules (1939b). La Douceur de la vie (Les Hommes de bonne volonté, Tome XVIII). Paris :

Flammarion.

ROMAINS, Jules (1939c). Cela dépend de vous. Paris : Flammarion.

ROMAINS, Jules (1940). Sept mystères du destin de l'Europe. New-York : Éditions de la Maison

française.

ROMAINS, Jules (1941). Messages aux Français. New-York : Éditions de la Maison française.

RomaIns, Jules (1946a). Le Tapis magique (Les Hommes de bonne volonté, Tome XXV). Paris :

Flammarion.

ROMAINS, Jules (1946b). Françoise (Les Hommes de bonne volonté, Tome XXVI). Paris : Flammarion.

ROMAINS, Jules (1946c). Le 7 octobre (Les Hommes de bonne volonté, Tome XXVII). Paris : Flammarion.

ROMAINS, Jules (1952). Saints de notre calendrier. Paris : Flammarion.

ROMAINS, Jules (1955). Passagers de cette planète. Paris : Grasset.

ROMAINS, Jules (1958). Souvenirs et confidences d'un écrivain. Paris : Fayard.

ROMAINS, Jules (1964). Ai-je fait ce que j'ai voulu?. Paris : Wesmael-Charlier.

ROMAINS, Jules (1982). Les Dossiers préparatoires des Hommes de bonne volonté : les tomes XV et XVI,

Prélude à Verdun et Verdun. Paris : Flammarion.

schakovsкoY, Zinaïda (2007). À la recherche de Nabokov. Paris : L'Âge d'homme.

TOUCHARD, Jean (2002). «En guise d'ouverture : littérature et politique. Faire de la politique sans le savoir ", in Denis-Constant Martin (éd.). Sur la piste des OPNI. Paris : Karthala, pp. 47-72.

\section{NOTES}

1. Il est d'ailleurs curieux de noter que Régis Antoine, dans son panorama de la littérature pacifiste et internationaliste française, 1915-1935 (Antoine, 2002), ne mentionne Jules Romains qu'en passant, et sans donner la moindre précision sur la nature et les moyens de son engagement.

2. Comme en témoigne Zinaïda Schakovskoy, Romains a usé systématiquement de son influence personnelle et institutionnelle pour sauver ceux qui étaient menacés par la barbarie nazie : « En collaboration avec le PEN club français et son président Jules Romains, nous procurions des visas à des Juifs allemands » (Schakovskoy, $2002: 45$ ).

\section{RÉSUMÉS}

Jules Romains n'a pas été au front, mais il n'a cessé d'agir et d'écrire contre la guerre. Outre ses nombreux essais en faveur de la paix et son long poème Europe, qui met les foules en demeure de refuser la guerre, il a raconté Verdun dans Les Hommes de bonne volonté. Il peint l'arrière, mais 
surtout les tranchées, et donne ainsi un véritable témoignage. C'est qu'il a consulté les archives publiques et intimes, et que son empathie de romancier lui permet de vivre avec intensité la vie des soldats ordinaires comme celle des généraux. Il ne veut pas cependant être de ces héros de l'arrière qui glorifient une guerre dont ils n'ont pas souffert. Il se dédouble donc : il a deux frères fictionnels, dont l'un reste à Paris, et dont l'autre part à Verdun. Cette dissociation entre l'individu et le poète en qui la psyché universelle se cristallise permet à Romains de faire résonner simultanément le chant qui célèbre le courage des soldats et le contre-chant qui déplore la misère de la guerre.

Jules Romains did not go to the front, but he made an unremitting effort to fight against war. He wrote numerous essays and a long poem entitled Europe, where he enjoins European peoples to say no to the war. He also recounted the Battle of Verdun in Men of Good Will. He evokes the life behind the lines, but also in the trenches, and he succeeds in bearing witness to the war experience. He consulted public and private archives, and his literary empathy permits him to live intensely the life of an enlisted man as well as the life of a general. However, he does not want to glorify a war he did not make. That is why he splits himself into two characters. He has two fictional brothers: the first one stays in Paris, the second one goes to Verdun. This dissociation between the ordinary individual and the poet who is able to crystallize the universal psyche allows Romains to cause both the hymn to the soldiers' courage and the elegy which deplores the war's atrocity to resonate.

INDEX

Keywords : Romains (Jules), pacifism, Verdun, account, fiction

Mots-clés : Romains (Jules), pacifisme, Verdun, témoignage, fiction

\section{AUTEUR}

\section{AUGUSTIN VOEGELE}

Université de Haute-Alsace

augustin.voegele@uha.fr 